Supporting Information

\title{
"H-type" Like Constructed Dimer, Another Way to Enhance the Thermally Activated Delayed Fluorescence Effect
}

\author{
Rongchuan Sü ${ }^{a} w^{\prime}$ and Zhenmei Huang $\%$
}

a Department of Pharmacology, North Sichuan Medical College, Nanchong, 637100, China

b College of Chemistry, Sichuan University, Chengdu, Sichuan, 610064, China

\section{Corresponding Author}

*srcxkai@nsmc.edu.cn

\section{Calculation Methods}

LE percentage and NTO analysis were performed by using the Multiwfn v.3.8 software package.

RDG picture was rendered by using VMD software. The $\omega_{\mathrm{eff}}$, and $\mathrm{S}_{\mathrm{eff}}$ were obtained by means of Eqs. (S1) and (S2) ${ }^{1}$.

$\hbar \omega_{e_{f f}}=\frac{\Sigma \hbar \omega_{j} S_{j}}{S_{e f f}}$

$S_{e_{f f}}=\sum S_{j}$

Molecular structure and $\Delta E_{S T}$ 
Ground-state $\left(\mathrm{S}_{0}\right)$ structures were optimized with B3LYP-D3(BJ) /def2-SVP level ${ }^{2}$, ${ }^{\text {, further }}$ calculations were employed to confirm that the structures were in stable states. Range separation functional, CAM-B3LYP was wieldy used in excited geometry optimization in TADF theoretical investigations. The B3LYP functional would overestimate electron delocalization in the excited states significantly, CAM-B3LYP would descript excited geometry more accurately in donoracceptor charge-transfer TADF molecules ${ }^{1,4}$. As mentioned above we choose CAM-B3LYP to optimize excited geometries. Excited-state configurations of $\mathrm{S}_{1}$ and $\mathrm{T}_{1}$ were optimized by CAMB3LYP-D3(BJ) function with def2-SVP basis set. $\mathrm{S}_{1}$-state geometry was optimized with TD-DFT method while $\mathrm{T}_{1}$-state geometry was obtained through spin-unrestricted calculation. All calculations were performed in toluene solution.

Range separation functional, $\omega$ B97XD or LC- $\omega$ PBE function with def2-SVP basis set within Tamm-Dancoff approximation (TDA) method may provide a better prediction of emission behavior within TADF research area ${ }^{4,5}$. The optimal $\omega$ parameters were determined by minimizing $s^{2}$

$$
J^{2}=\sum_{i=0}^{1}\left[\varepsilon_{\mathrm{H}}(N+i)+\mathrm{IP}(N+i)\right]^{2}
$$

where IP and $\varepsilon_{H}$ denote the ionization potential and HOMO energy, respectively, and $N$ is the number of electrons ${ }^{6}$. The optimal $\omega$ values were listed in Table S11. Reverse intersystem crossing rate

Reverse intersystem crossing rates were calculated through the Fermi Golden rule ${ }^{7,8}$ :

$$
k_{\mathrm{RISC}}=\frac{2 \pi}{\hbar} \rho_{\mathrm{FC}}\left|\left\langle S_{1}\left|\hat{H}_{s o}\right| T_{1}\right\rangle\right|^{2}
$$


where the $\left\langle S_{1}\left|\hat{H}_{s o}\right| T_{1}\right\rangle$ is the calculated SOC constants, $\hbar$ denotes the reduced Planck constant, and $\rho_{\mathrm{FC}}$ represents the Franck-Condon-weighted density. The $\rho_{\mathrm{FC}}$ value can be determined through Marcus-Levich-Jortner theory9, 10:

$$
\rho_{\mathrm{FC}}=\frac{1}{\sqrt{4 \pi \lambda_{\mathrm{M}} k_{\mathrm{B}} T}} \sum_{n=0}^{\infty} \exp (-S) \frac{S_{n !}^{n}}{n !} \exp \left[-\frac{\left(\Delta E_{\mathrm{ST}}+n \hbar \omega_{\mathrm{eff}}+\lambda_{\mathrm{M}}\right)^{2}}{4 \lambda_{\mathrm{M}} k_{\mathrm{B}} T}\right]
$$

where $\lambda_{\mathrm{M}}$ represents the reorganization energy according to Marcus theory; $k_{\mathrm{B}}$ denotes the Boltzmann constant; T represents the temperature, $S$ represents the effective Huang-Rhys factor which calculated through Dushin program ${ }^{11}, \hbar \omega_{\text {eff }}$ denotes the effective energy of a model representing the nonclassical high-frequency intramolecular vibrations. The $k_{\mathrm{RISC}}$ values were computed with the triplet geometry.

\section{Spin-orbit coupling constants}

The SOC constants between first excited singlet state $\left(\mathrm{S}_{1}\right)$ and first excited triplet states $\left(\mathrm{T}_{1}\right)$, $\left\langle\mathrm{S}_{1}\left|\hat{\mathrm{H}}_{\mathrm{SO}}\right| \mathrm{T}_{1}\right\rangle$, was calculated by considering three $\mathrm{T}_{1}$ sub-states $(\mathrm{m}=0, \pm 1)$.

$$
\left\langle S_{1}\left|\hat{H}_{s o}\right| T_{1}\right\rangle=\sqrt{\sum_{m}\left\langle S_{1}\left|\hat{H}_{s o}\right| T_{1, m}\right\rangle}
$$

The spin-orbit coupling operator based on Breit-Pauli spin-orbit Hamiltonian with effective charge approximations are given as ${ }^{12,13}$ :

$$
\begin{aligned}
& \hat{H}_{s o}^{x}=1 / 2 \sum_{p q} h_{P q}^{x}\left(a_{p \alpha}^{\dagger} a_{q \beta}+a_{p \beta}^{\dagger} a_{q \alpha}\right) \\
& \hat{H}_{s o}^{y}=1 /(2 i) \sum_{p q} h_{P q}^{y}\left(a_{p \alpha}^{\dagger} a_{q \beta}-a_{p \beta}^{\dagger} a_{q \alpha}\right) \\
& \hat{H}_{s o}^{z}=1 / 2 \sum_{p q} h_{P q}^{z}\left(a_{p \alpha}^{\dagger} a_{q \alpha}-a_{p \beta}^{\dagger} a_{q \beta}\right)
\end{aligned}
$$


where the creation $\left\{a_{p \alpha}^{\dagger} \ldots\right\}$ and annihilation $\left\{a_{q \beta} \ldots\right\}$ operators obey the commutation relation \{

$\left.a_{p \sigma}^{\dagger}, a_{q \sigma^{\prime}}\right\}=\delta_{p q} \delta_{\sigma \sigma^{\prime}}$. The matrix element $h_{P q}^{d}$ of the SOC Hamiltonian between Kohn-Sham orbitals is defined as:

$$
h_{p q}^{d} \equiv\left\langle\psi_{p}\left|\zeta(r) \hat{l}_{d}\right| \psi_{q}\right\rangle, d \in\{x, \mathrm{y}, \mathrm{z}\} \quad \text { S8 }
$$

The calculated SOC constants were based on TD-DFT calculation. All SOC constant calculations were employed with PySOC software ${ }^{12}$.

\section{Experiment methods}

Powder X-ray diffraction (XRD) pattern was taken by using X Pert Pro MPD X-ray diffractometer with a $\mathrm{Cu}-\mathrm{K} \alpha$ radiation source $(\lambda=1.54056 \AA, 40 \mathrm{KV}, 35 \mathrm{~mA})$. XRD pattern of single crystal was obtained from Mercury software.

\section{Calculation results}

Table S1. Calculated gibbs free energy difference between monomer and dimer.

\begin{tabular}{cc}
\hline Molecule & $\Delta \mathrm{G}$ (hartree) \\
\hline DMAC-BPI & 0.050909 \\
SDMAC-BPI & 0.022984 \\
QA-PCN & 0.027683 \\
\hline
\end{tabular}

Table S2. Calculated gibbs free energy.

\begin{tabular}{ccc}
\hline Molecule & State & $\mathrm{G}$ (hartree) \\
\hline \multirow{3}{*}{ DMAC-BPI } & $p$-form & -2398.595504 \\
& $m$-form & -2398.595504 \\
SDMAC-BPI & $p$-form & -4797.241917 \\
& -1764.996229
\end{tabular}




\begin{tabular}{ccc} 
& $m$-form & -1764.995322 \\
& dimer & -3530.014535 \\
QA-PCN & $p$-form & -1296.198058 \\
& $m$-form & -1296.198058 \\
& dimer & -2592.423799 \\
\hline
\end{tabular}

Table S3. Summary photophysical properties of doped films (PMMA) with various concentrations.

\begin{tabular}{|c|c|c|c|c|c|c|c|}
\hline $\begin{array}{c}\text { Concentration } \\
(\%)\end{array}$ & $\begin{array}{l}\lambda_{\text {abs }}{ }^{a)} \\
{[\mathrm{nm}]}\end{array}$ & $\begin{array}{l}\lambda_{\text {em }} \text { b) } \\
(\mathrm{nm})\end{array}$ & $\begin{array}{l}\Phi_{\text {PL }} \\
(\%)\end{array}$ & $\begin{array}{c}\tau_{p} \\
(\mathrm{~ns})\end{array}$ & $\begin{array}{c}\tau_{\mathrm{d}} \\
(\mu \mathrm{s})\end{array}$ & $\begin{array}{l}R d \\
(\%)\end{array}$ & $\begin{array}{c}k_{\text {RISC }} \\
\left(1^{6} s^{-1}\right)\end{array}$ \\
\hline 5 & 411 & 410 & 56.23 & 23.67 & 3.86 & 65.27 & 0.75 \\
\hline 10 & 409 & 422 & 66.05 & 25.33 & 3.53 & 63.49 & 0.78 \\
\hline 20 & 406 & 430 & 72.10 & 27.22 & 3.01 & 66.37 & 0.99 \\
\hline 50 & 404 & 439 & 75.12 & 26.79 & 2.66 & 64.76 & 1.07 \\
\hline 90 & 396 & 449 & 77.76 & 26.28 & 2.29 & 62.25 & 1.16 \\
\hline
\end{tabular}

a) lowest energy of absorption, b) onset of emission

Table S4. Calculated emission energies (E) of $\mathrm{S}_{1}, \mathrm{~T}_{1}$ states, and oscillator strength $(f)$ of monomer with various TD(A)-DFT methods with def2-SVP basis set.

\begin{tabular}{llllllll}
\hline DFT function & $\mathrm{E}\left(\mathrm{S}_{1}\right)$ & $\lambda\left(\mathrm{S}_{1}\right)$ & $\mathrm{RE})$ & $f$ & $\mathrm{E}\left(\mathrm{T}_{1}\right)$ & $\lambda\left(\mathrm{T}_{1}\right)$ & $\left.\mathrm{RE}^{\mathrm{b}}\right)$ \\
& $(\mathrm{eV})$ & $(\mathrm{nm})$ & $(\%)$ & $\left(10^{-2}\right)$ & $(\mathrm{eV})$ & $(\mathrm{nm})$ & $(\%)$ \\
\hline CAM-B3LYP & 3.37 & 367.95 & +15.02 & 0.01 & 2.61 & 475.10 & -10.31 \\
B3LYP & 2.37 & 523.21 & +19.11 & 0.01 & 2.52 & 492.06 & -13.40 \\
PBE0 & 2.51 & 494.02 & +14.33 & 0.01 & 2.49 & 497.99 & -14.43 \\
M06-HF & 4.12 & 300.97 & +40.61 & 0.20 & 3.39 & 365.78 & +16.49 \\
M06-2X & 3.33 & 372.37 & +13.65 & 0.01 & 3.01 & 411.96 & +3.43 \\
$\omega$ 397XD & 3.09 & 401.29 & +5.46 & 0.01 & 2.77 & 447.65 & -4.81 \\
$\omega$ 397XD ${ }^{\mathrm{a})}$ & 3.09 & 401.29 & +5.46 & 0.03 & 2.96 & 418.92 & +1.72 \\
LC- $\omega$ PBE & 5.44 & 227.94 & +85.67 & 1.89 & 5.09 & 243.61 & +74.91 \\
LC- $\omega$ PBE & 5.47 & 226.69 & +86.69 & 2.11 & 5.09 & 243.61 & +74.91 \\
\hline
\end{tabular}

a) TDA methods, b) Relative error 
Table S5. Calculated emission energies (E) of $\mathrm{S}_{1}, \mathrm{~T}_{1}$ states, and oscillator strength $(f)$ of monomer with various TD-DFT/6-31G(d) methods for DMAC-BPI.

\begin{tabular}{|c|c|c|c|c|c|c|c|}
\hline Functional & $\begin{array}{c}E\left(S_{1}\right) \\
(e V)\end{array}$ & $\begin{array}{l}\lambda\left(\mathbf{S}_{1}\right) \\
(\mathbf{n m})\end{array}$ & $\begin{array}{l}\text { RE } \\
(\%)\end{array}$ & $\begin{array}{c}f \\
\left(10^{-2}\right) \\
\end{array}$ & $\begin{array}{c}\mathbf{E}\left(\mathbf{T}_{1}\right) \\
(\mathrm{eV})\end{array}$ & $\begin{array}{l}\lambda\left(\mathbf{T}_{1}\right) \\
(\mathbf{n m})\end{array}$ & $\begin{array}{l}\text { RE } \\
(\%)\end{array}$ \\
\hline CAM-B3LYP & 3.31 & 374.62 & +12.97 & 0.00 & 2.56 & 484.38 & -12.03 \\
\hline B3LYP & 2.29 & 541.48 & -21.84 & 0.01 & 2.44 & 508.20 & -16.15 \\
\hline PBE0 & 2.43 & 510.29 & -17.06 & 0.01 & 2.43 & 510.29 & -16.49 \\
\hline M06-2X & 3.29 & 376.90 & +12.29 & 0.00 & 2.98 & 416.11 & +2.41 \\
\hline$\omega B 97 X D$ & 3.03 & 409.24 & +3.41 & 0.00 & 2.71 & 457.56 & -6.87 \\
\hline LC- $\omega$ PBE & 5.52 & 224.64 & +88.40 & 1.86 & 5.10 & 243.14 & +75.26 \\
\hline
\end{tabular}

Table S6. Calculated emission energies (E) of $\mathrm{S}_{1}, \mathrm{~T}_{1}$ states, and oscillator strength $(f)$ of monomer with various TD-DFT/6-311G(d) methods for DMAC-BPI.

\begin{tabular}{|c|c|c|c|c|c|c|c|}
\hline Functional & $\begin{array}{c}\mathbf{E}\left(\mathbf{S}_{1}\right) \\
(\mathrm{eV})\end{array}$ & $\lambda\left(\mathbf{S}_{1}\right)(\mathrm{nm})$ & $\begin{array}{l}\text { RE } \\
(\%)\end{array}$ & $\begin{array}{c}f \\
\left(10^{-2}\right)\end{array}$ & $E\left(T_{1}\right)(e V)$ & $\lambda\left(\mathrm{T}_{1}\right)(\mathrm{nm})$ & RE (\%) \\
\hline CAM-B3LYP & 3.28 & 378.05 & +11.95 & 0.00 & 2.55 & 486.27 & -12.37 \\
\hline B3LYP & 2.28 & 543.86 & -22.18 & 0.01 & 2.43 & 510.29 & -16.49 \\
\hline PBE0 & 2.43 & 510.29 & -17.06 & 0.01 & 2.42 & 512.40 & -16.84 \\
\hline M06-2X & 3.29 & 376.90 & +12.29 & 0.00 & 2.95 & 420.34 & +1.37 \\
\hline$\omega \mathrm{B} 97 \mathrm{XD}$ & 3.00 & 413.33 & +2.39 & 0.00 & 2.70 & 459.26 & -7.22 \\
\hline LC- $\omega$ PBE & 5.40 & 229.63 & +84.30 & 1.00 & 5.04 & 246.03 & +73.20 \\
\hline
\end{tabular}

Table S7. Calculated emission energies (E) of $\mathrm{S}_{1}, \mathrm{~T}_{1}$ states, and oscillator strength $(f)$ of monomer with various TD-DFT/6-31+G(d) methods for DMAC-BPI.

\begin{tabular}{cccccccc}
\hline Functional & $\mathbf{E}\left(\mathbf{S}_{\mathbf{1}}\right)$ & $\lambda\left(\mathbf{S}_{\mathbf{1}}\right)(\mathbf{n m})$ & $\mathbf{R E}(\mathbf{\%})$ & $\begin{array}{c}\boldsymbol{f} \\
(\mathbf{1 0})\end{array}$ & $\begin{array}{c}\mathbf{E}\left(\mathbf{T}_{\mathbf{1}}\right) \\
(\mathbf{e V})\end{array}$ & $\boldsymbol{\lambda}\left(\mathbf{T}_{\mathbf{1}}\right)(\mathbf{n m})$ & $\mathbf{R E}(\mathbf{\%})$ \\
\hline CAM- & 3.27 & 379.20 & +11.60 & 0.00 & 2.58 & 480.62 & -11.34 \\
B3LYP & & & & & & & \\
B3LYP & 2.28 & 543.86 & -22.18 & 0.00 & 2.45 & 506.12 & -15.81 \\
PBE0 & 2.43 & 510.29 & -17.06 & 0.01 & 2.44 & 508.20 & -16.15 \\
M06-2X & 3.27 & 379.20 & +11.60 & 0.00 & 2.98 & 416.11 & +2.41 \\
$\omega$ B97XD & 2.97 & 417.51 & +1.37 & 0.00 & 2.72 & 455.88 & -6.53 \\
LC- $\omega$ PBE & 4.38 & 283.11 & +49.49 & 0.38 & 4.30 & 288.37 & +47.77 \\
\hline
\end{tabular}


Table S8. Calculated emission energies (E) of $\mathrm{S}_{1}, \mathrm{~T}_{1}$ states, and oscillator strength $(f)$ of monomer with various TDA-DFT/6-31G(d) methods for DMAC-BPI.

\begin{tabular}{|c|c|c|c|c|c|c|c|}
\hline Functional & $\begin{array}{c}\mathbf{E}\left(\mathbf{S}_{1}\right) \\
(\mathrm{eV})\end{array}$ & $\begin{array}{l}\lambda\left(\mathbf{S}_{1}\right) \\
(\mathbf{n m})\end{array}$ & $\begin{array}{l}\text { RE } \\
(\%)\end{array}$ & $\begin{array}{c}f \\
\left(10^{-2}\right)\end{array}$ & $\begin{array}{c}\mathbf{E}\left(\mathbf{T}_{1}\right) \\
(\mathrm{eV})\end{array}$ & $\begin{array}{l}\lambda\left(\mathbf{T}_{1}\right) \\
(\mathbf{n m})\end{array}$ & $\begin{array}{l}\mathrm{RE} \\
\text { (\%) }\end{array}$ \\
\hline CAM-B3LYP & 3.31 & 374.62 & +12.97 & 0.00 & 2.95 & 420.34 & +1.37 \\
\hline B3LYP & 2.29 & 541.48 & -21.84 & 0.01 & 2.53 & 490.12 & -13.06 \\
\hline PBE0 & 2.44 & 508.20 & -16.72 & 0.01 & 2.58 & 480.62 & -11.34 \\
\hline M06-2X & 3.29 & 376.90 & +12.29 & 0.00 & 3.10 & 400.00 & +6.53 \\
\hline$\omega B 97 X D$ & 3.03 & 409.24 & +3.41 & 0.00 & 2.90 & 427.59 & -0.34 \\
\hline LC- $\omega$ PBE & 5.55 & 223.42 & +89.42 & 2.13 & 5.10 & 243.14 & +75.26 \\
\hline
\end{tabular}

Table S9. Calculated emission energies (E) of $\mathrm{S}_{1}, \mathrm{~T}_{1}$ states, and oscillator strength $(f)$ of monomer with various TDA-DFT/6-311G(d) methods for DMAC-BPI.

\begin{tabular}{|c|c|c|c|c|c|c|c|}
\hline Functional & $\begin{array}{c}\mathbf{E}\left(\mathbf{S}_{1}\right) \\
(\mathrm{eV})\end{array}$ & $\lambda\left(\mathbf{S}_{1}\right)(\mathrm{nm})$ & $\begin{array}{l}\text { RE } \\
(\%)\end{array}$ & $\begin{array}{c}f \\
\left(10^{-2}\right)\end{array}$ & $E\left(T_{1}\right)(e V)$ & $\lambda\left(\mathrm{T}_{1}\right)(\mathrm{nm})$ & RE (\%) \\
\hline CAM-B3LYP & 3.29 & 376.90 & +12.29 & 0.00 & 2.93 & 423.21 & +0.69 \\
\hline B3LYP & 2.28 & 543.86 & -22.18 & 0.01 & 2.51 & 494.02 & -13.75 \\
\hline PBE0 & 2.43 & 510.29 & -17.06 & 0.01 & 2.57 & 482.49 & -11.68 \\
\hline M06-2X & 3.29 & 376.90 & +12.29 & 0.00 & 3.09 & 401.29 & +6.19 \\
\hline$\omega \mathrm{B} 97 \mathrm{XD}$ & 3.00 & 413.33 & +2.39 & 0.00 & 2.88 & 430.56 & -1.03 \\
\hline LC- $\omega$ PBE & 5.42 & 228.78 & +84.98 & 0.98 & 5.04 & 246.03 & +73.20 \\
\hline
\end{tabular}

Table S10. Calculated emission energies (E) of $\mathrm{S}_{1}, \mathrm{~T}_{1}$ states, and oscillator strength $(f)$ of monomer with various TDA-DFT/6-31+G(d) methods for DMAC-BPI.

\begin{tabular}{cccccccc}
\hline Functional & $\begin{array}{c}\mathbf{E}\left(\mathbf{S}_{\mathbf{1}}\right) \\
(\mathbf{e V})\end{array}$ & $\left.\boldsymbol{\lambda}\left(\mathbf{S}_{\mathbf{1}}\right) \mathbf{( n m}\right)$ & $\mathbf{R E}(\mathbf{\%})$ & $\begin{array}{c}\boldsymbol{f} \\
\left(\mathbf{1 0}^{-2}\right)\end{array}$ & $\begin{array}{c}\mathbf{E}\left(\mathbf{T}_{\mathbf{1}}\right) \\
(\mathbf{e V})\end{array}$ & $\boldsymbol{\lambda}\left(\mathbf{T}_{\mathbf{1}}\right)(\mathbf{n m})$ & $\mathbf{R E}(\mathbf{\%})$ \\
\hline CAM- & 3.28 & 378.05 & +11.95 & 0.00 & 2.95 & 420.34 & +1.37 \\
B3LYP & & & & & & & \\
B3LYP & 2.28 & 543.86 & -22.18 & 0.01 & 2.53 & 490.12 & -13.06 \\
PBE0 & 2.43 & 510.29 & -17.06 & 0.01 & 2.59 & 478.76 & -11.00 \\
M06-2X & 3.27 & 379.20 & +11.60 & 0.00 & 3.10 & 400.00 & +6.53 \\
$\omega B 97 X D$ & 2.97 & 417.51 & +1.37 & 0.00 & 2.89 & 429.07 & -0.69 \\
LC- $\omega$ PBE & 4.39 & 282.46 & +49.83 & 0.62 & 4.31 & 287.70 & +48.11 \\
\hline
\end{tabular}


Table S11. Optimal $\omega$ values for monomer and dimer with various DFT methods.

\begin{tabular}{cccc}
\hline Molecule & Functional & Basis set & $\omega$ \\
\hline & & def2-SVP & 0.1273 \\
& $\omega B 97 X D$ & $6-31 G(d)$ & 0.1284 \\
& & $6-311 G(d)$ & 0.1246 \\
DMAC-BPI $^{1}$ & & $6-31+\mathrm{G}(\mathrm{d})$ & 0.1210 \\
\cline { 2 - 4 } & & def2-SVP & 0.1639 \\
& & $6-31 \mathrm{G}(\mathrm{d})$ & 0.1660 \\
& & $6-311 \mathrm{G}(\mathrm{d})$ & 0.1615 \\
DMAC-BPI $^{2}$ & $\omega \mathrm{B} 97 \mathrm{XD}$ & $\mathrm{def} 2-\mathrm{SVP}$ & 0.1046 \\
SDMAC-BPI $^{1}$ & $\omega \mathrm{B} 97 \mathrm{XD}$ & $\mathrm{def} 2-\mathrm{SVP}$ & 0.1375 \\
SDMAC-BPI $^{2}$ & $\omega \mathrm{B} 97 \mathrm{XD}$ & def2-SVP & 0.1194 \\
\hline
\end{tabular}

${ }^{1}$ monomer, ${ }^{2}$ dimer.
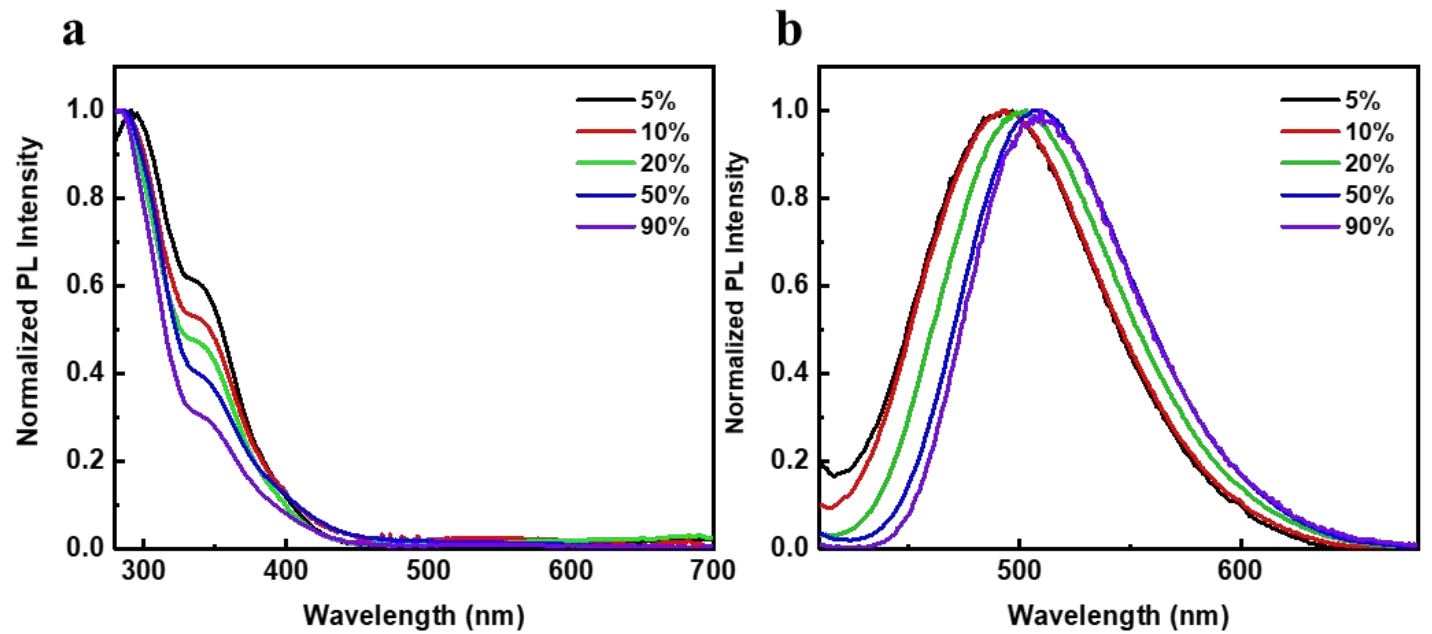

Figure S1. Normalized a) absorption, b) emission spectrum for doped films with different concentration. 

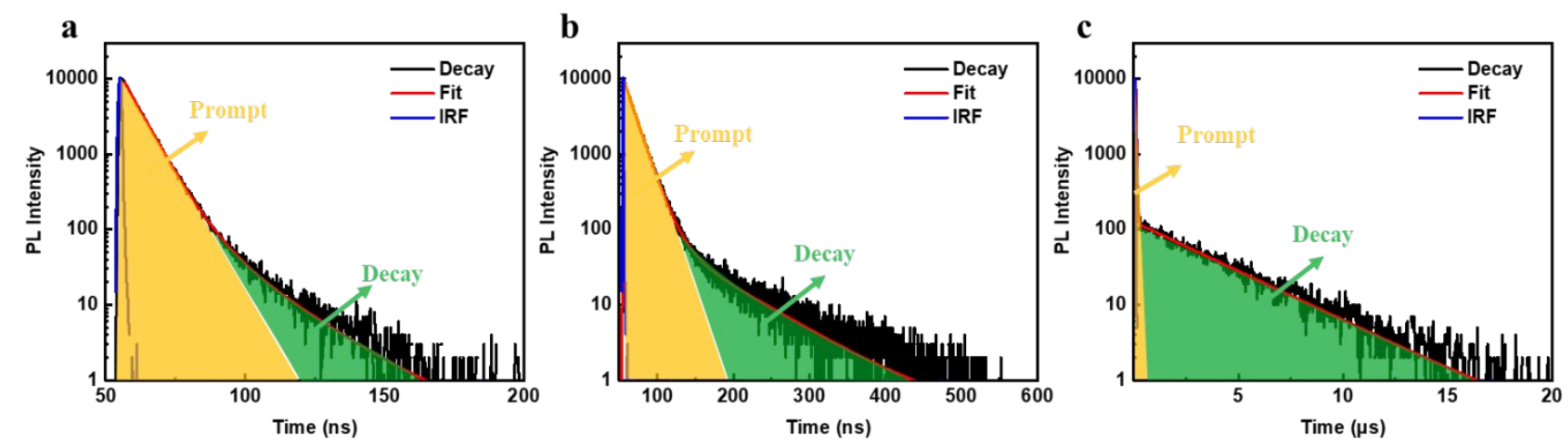

Figure S2. PL transient decay curves of DMAC-BPI in toluene solution $\left(10^{-5} \mathrm{M}\right)$ after a) $\mathrm{O}_{2}$ b) air and c) $\mathrm{N}_{2}$ bubbled.
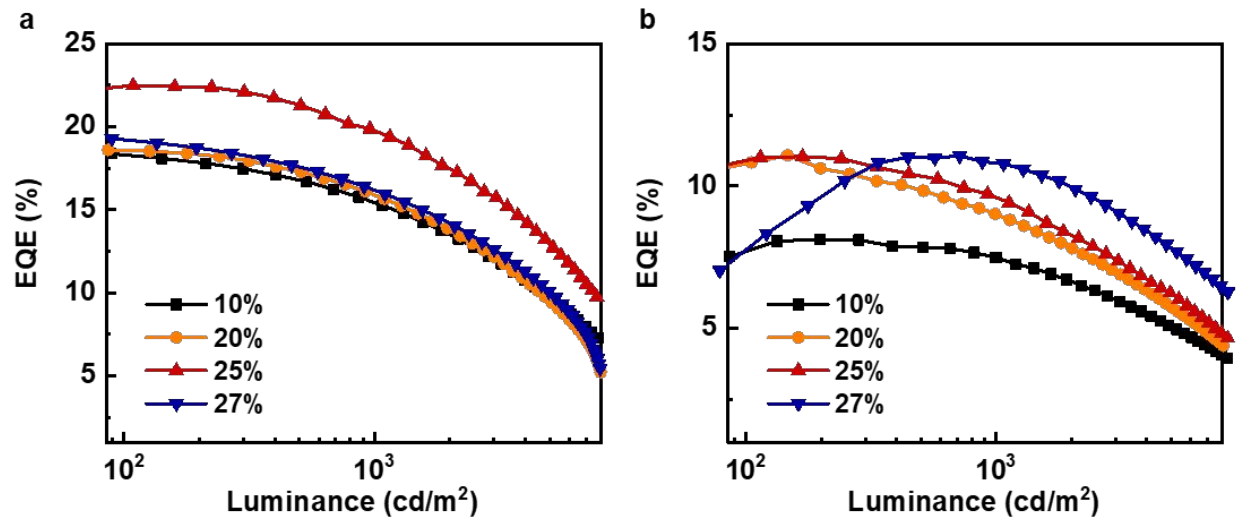

Figure S3. EQE versus luminance curves of doped OLED devices for a) ITO/TAPC (30 $\mathrm{nm}) / \mathrm{TCTA}(10 \mathrm{~nm}) / \mathrm{DMAC}-\mathrm{DPS}: x \mathrm{wt} \%$ DMAC-BPI $(30 \mathrm{~nm}) / \mathrm{DPEPO}(10 \mathrm{~nm}) / \mathrm{BPhen}(30$ $\mathrm{nm}) / \mathrm{LiF}(0.8 \mathrm{~nm}) / \mathrm{Al}$, and b) ITO/HAT-CN (5 nm)/NPB (30 nm)/DMAC-DPS: $x \mathrm{wt} \% m$ CBP (30 $\mathrm{nm}) / \mathrm{Tmpypb}(30 \mathrm{~nm}) \mathrm{LiF}(0.8 \mathrm{~nm}) / \mathrm{Al}$ 


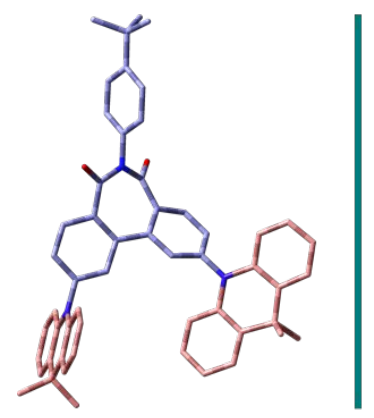

monomer

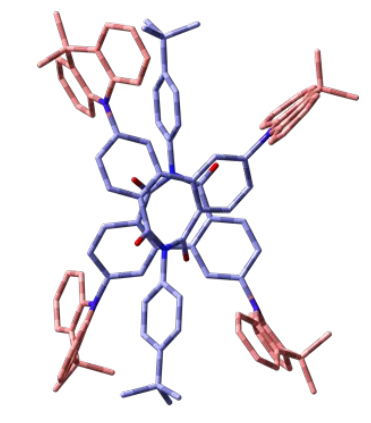

dimer

Figure S4. Donor (light red) and Acceptor (light blue) parts defined for LE percentage calculation for DMAC-BPI.

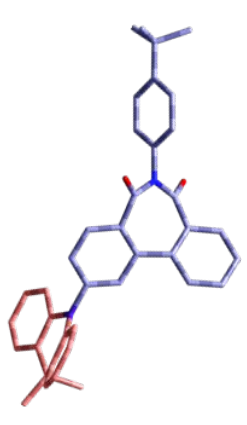

monomer

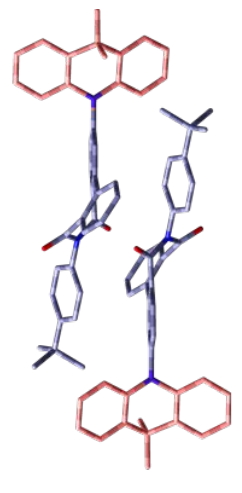

dimer

Figure S5. Donor (light red) and Acceptor (light blue) parts defined for LE percentage calculation for SDMAC-BPI.

a

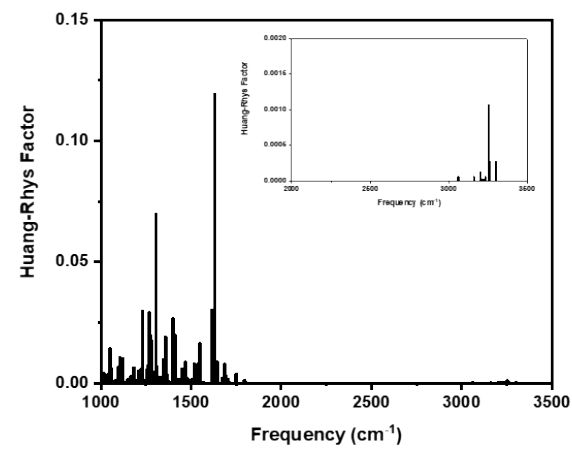

b

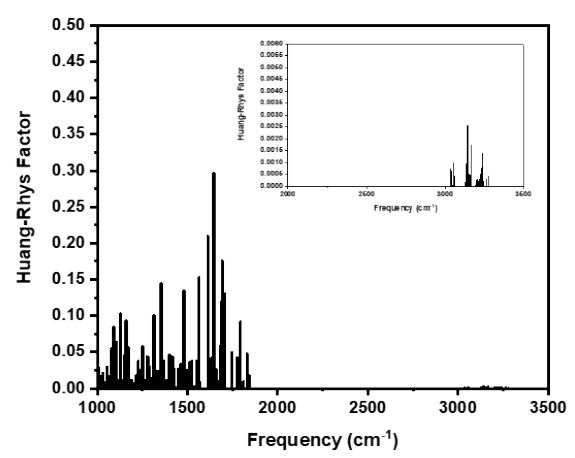


Figure S6. Huang-Rhys factors associated with the high-frequency vibrational modes for DMAC-BPI, a) monomer and b) dimer.

a

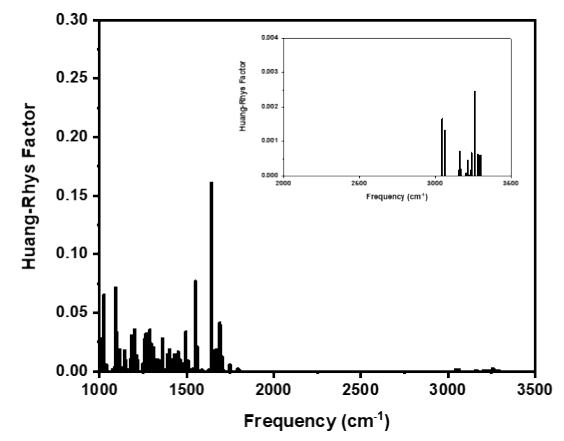

b

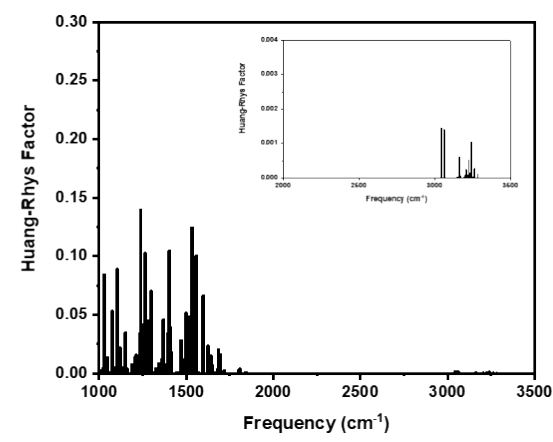

Figure S7. Huang-Rhys factors associated with the high-frequency vibrational modes for SDMAC-BPI, a) monomer and b) dimer.

Front

Top

Side

$\mathbf{a}$
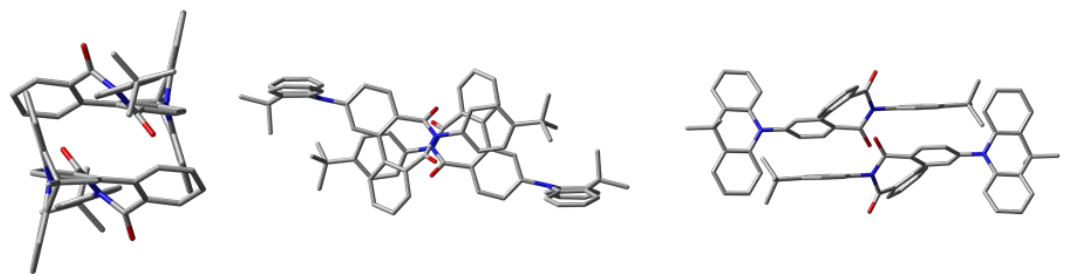

b
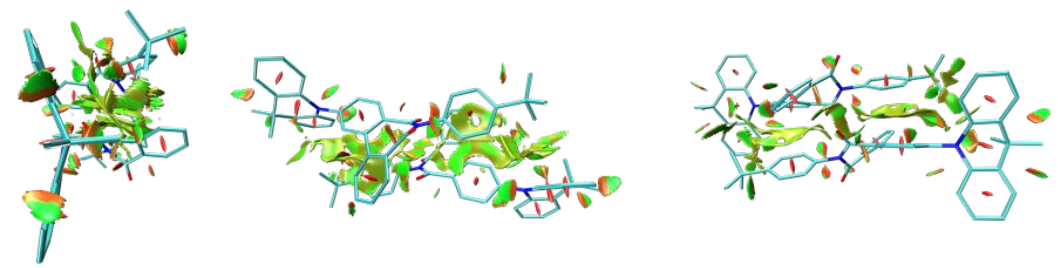

Figure S8. a) Three-view drawing structure of SDMAC-BPI and b) reduced density gradient (RDG) isosurface map with an isovalue of 0.5 for "H-type" like dimer.

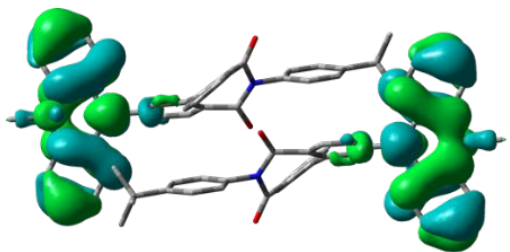

HOMO

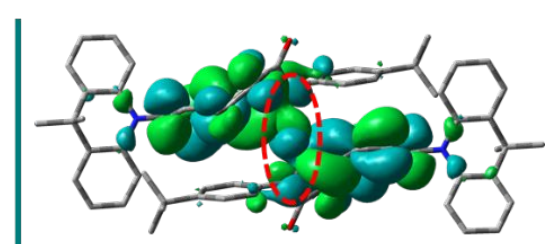

LUMO

Figure S9. HOMO/LUMO distribution of "H-type" like dimer (SDMAC-BPI). 


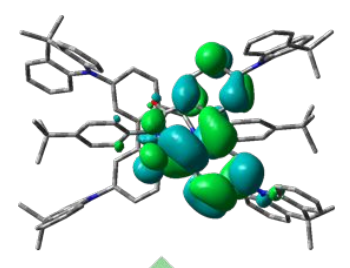

\section{8\%}

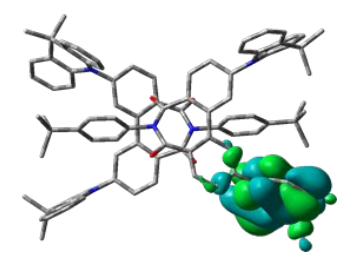

Figure S10. NTO analysis of $\mathrm{S}_{1}$ states for "H-type" like dimer(DMAC-BPI).

$\mathbf{a}$

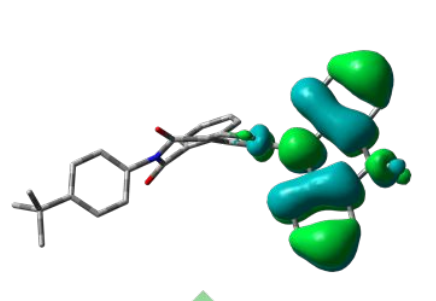

$95.9 \%$

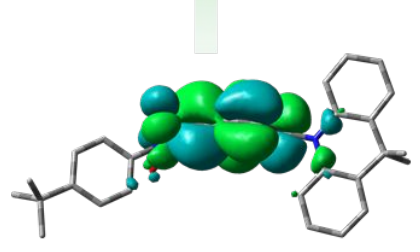

b

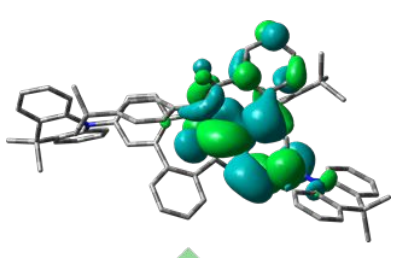

$94.3 \%$

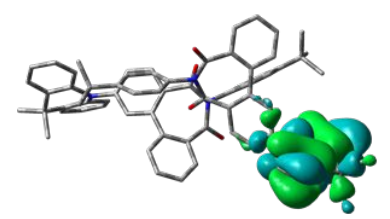

Figure S11. NTO analysis of $\mathrm{S}_{1}$ states for SDMAC-BPI, a) monomer and b) "H-type" like dimer. 
$\mathbf{a}$

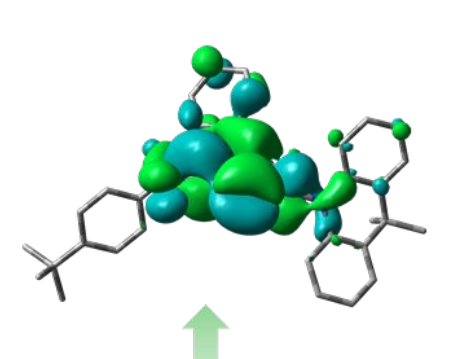

93.3\%

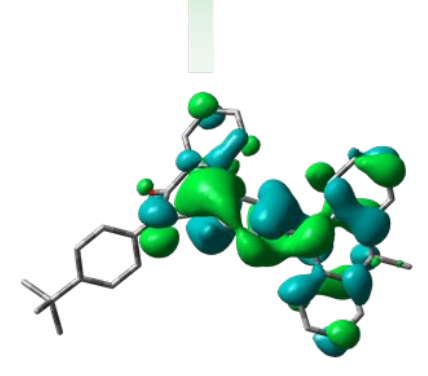

b

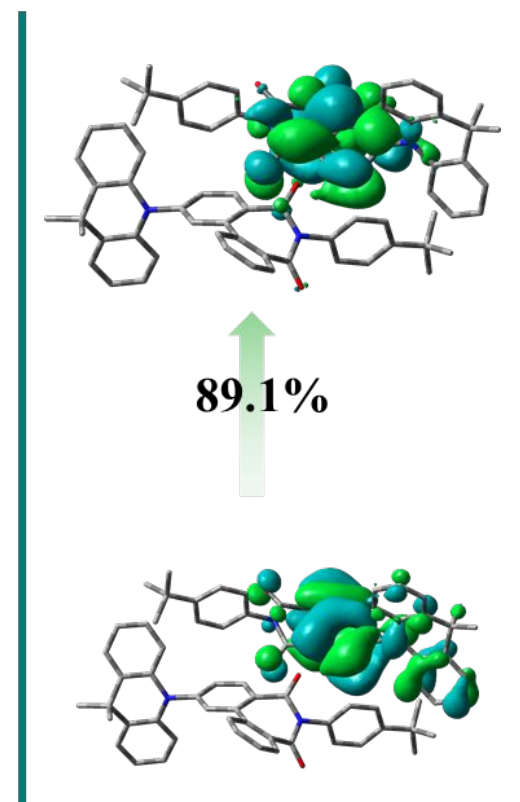

Figure S12 NTO analysis of $\mathrm{T}_{1}$ states for SDMAC-BPI, a) monomer and b) "H-type" like

\section{Discuss for calculation methods chosen processes}

The accuracy of TD(A)-DFT calculations depends on the selected methods. There is no universal method to predict TADF properties through TD(A)-DFT calculation. In this paper, we focus on the difference between monomer and dimer, the physical properties of monomer were known, so, we have tested numerous TD(A)-DFT methods and choose the methods which were in line with experimental data. As shown in Table S3 various functionals were verified in excited geometries, although CAM-B3LYP descript excited geometry more accurately in donor-acceptor chargetransfer TADF molecules, the prediction accuracy of $S_{1}$ and $T_{1}$ energy is far away from experimental data. The calculated energy of $\mathrm{S}_{1}$ is $3.37 \mathrm{eV}$ which afford a $15.02 \%$ relative error (RE). We have tested B3LYP and PBE0 functionals sequentially, both of them could not provide 
a satisfactory result, the minimal RE was $13.40 \%$. The M06-2X functional could afford a usable $\mathrm{T}_{1}$ energy calculation, $3.01 \mathrm{eV}$ which was similar to experimental data $(2.91 \mathrm{eV})^{14}$, but the prediction accuracy of $S_{1}$ was as poor as previous tested methods. The optimally tuned range separated DFT method was the last hope for our calculations. The $\omega$ B97XD and LC- $\omega$ PBE were tested sequentially. The $\omega$ B97XD functional could provide unified and precise results, the maximal RE was $5.46 \%$, the minimal RE was $1.72 \%$ for phosphorescence simulation with a TDA method. Even though the LC- $\omega$ PBE functional could afford some accurate results in some cases, but it not a suitable one in this calculation. As shown in Table S6 - Table S10 pople basis sets with various DFT functionals were examined, $\omega \mathrm{B} 97 \mathrm{XD}$ functional with $6-31+\mathrm{G}(\mathrm{d})$ basis set could afford the best accuracy of emission energy among the tests, but the $f$ value is zero, which is not consistent with experimental results. As a consequence, $\omega$ B97XD functional with def2-SVP basis set with TDA method was chosen in the further calculation.

To further prove that the DFT method we have chosen was suitable for other calculations, Natural transition orbitals (NTO) analysis were performed. As shown in Figure S13, the monomer performed a ${ }^{1} \mathrm{CT}$ (charge transfer) and ${ }^{3} \mathrm{CT}$ state. The holes were mainly located around the donor area and the particles were distributed at the acceptor region. Based on our former research ${ }^{14}$, both fluorescence and phosphorescence emission possessed a broad and featureless spectrum which mean a CT state. The same results of emission energy and pattern between calculation and 
experiment test, both proven that the method we chosen was suitable to describe the properties of these molecules.
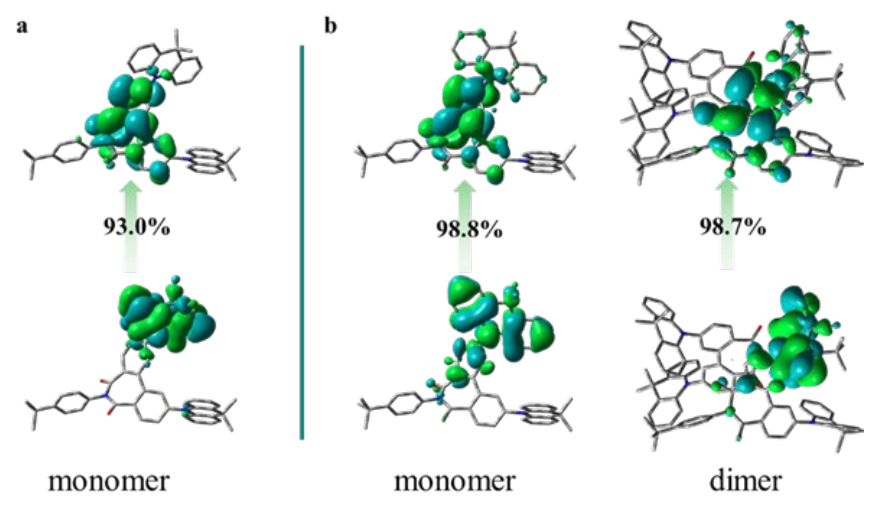

Figure S13. NTO analysis of a) $\mathrm{S}_{1}$ and b) $\mathrm{T}_{1}$ states for DMAC-BPI, monomer and "H-type" like dimer.

\section{Reference}

1. Samanta, P. K.; Kim, D.; Coropceanu, V.; Bredas, J. L., Up-Conversion Intersystem Crossing Rates in Organic Emitters for Thermally Activated Delayed Fluorescence: Impact of the Nature of Singlet vs Triplet Excited States. J. Am. Chem. Soc. 2017, 139, 4042-4051.

2. Zhang, Q.; Sun, S. Q.; Liu, W.; Leng, P. P.; Lv, X. L.; $\quad$ Wang, Y. X.; Chen, H. T.; Ye, S. F.; Zhuang, S. Q.; Wang, L., Integrating TADF Luminogens with AIE Characteristics Using A Novel Acridine-carbazole Gybrid as Donor for High-performance and Low Efficiency Roll-off OLEDs. J. Mater. Chem. C2019, 7, 9487-9495. 
3. Yang, T.; Liang, B.; Cheng, Z.; Li, C.; Lu, G.; Wang, Y., Construction of Efficient Deep-Red/Near-Infrared Emitter Based on A Large $\pi$-Conjugated Acceptor and Delayed Fluorescence OLEDs with External Quantum Efficiency of over 20\%. J. Phys. Chem. C 2019, $123,18585-18592$.

4. Sun, H.; Zhong, C.; Bredas, J. L., Reliable Prediction with Tuned Range-Separated Functionals of the Singlet-Triplet Gap in Organic Emitters for Thermally Activated Delayed Fluorescence. J. Chem. Theory Comput. 2015, 11, 3851-3858.

5. Woo, S. J.; Kim, Y.; Kim, Y. H.; Kwon, S. K.; Kim, J. J., A Spiro-silafluorenephenazasiline Donor-based Efficient Blue Thermally Activated Delayed Fluorescence Emitter and Its Host-dependent Device Characteristics. J. Mater. Chem. C 2019, 7, 4191-4198.

6. Tsuchiya, Y.; $\quad$ Ishikawa, Y.; $\quad$ Lee, S. H.; Chen, X. K.; $\quad$ Bredas, J. L.; $\quad$ Nakanotani, H.; Adachi, C., Thermally Activated Delayed Fluorescence Properties of Trioxoazatriangulene Derivatives Modified with Electron Donating Groups. Adv. Opt. Mater. 2021, 9, 2002174.

7. Robinson, G. W.; Frosch, R. P., Electronic Excitation Transfer and Relaxation. J. Chem. Phys. 1963, 38, 1187-1203.

8. Lawetz, V.; Orlandi, G.; Siebrand, W., Theory of Intersystem Crossing in Aromatic Hydrocarbons. J. Chem. Phys. 1972, 56, 4058-4072. 
9. Paul, L.; Moitra, T.; Ruud, K.; Chakrabarti, S., Strong Duschinsky Mixing Induced Breakdown of Kasha's Rule in an Organic Phosphor. J. Phys. Chem. Lett. 2019, 10, 369-374.

10. de Melo Neto, C. A. M.; Pereira, M. L.; Ribeiro, L. A.; Roncaratti, L. F.; da Silva Filho, D. A., Theoretical Prediction of Electron Mobility in Birhodanine Crystals and Their Sulfur Analogs. Chem. Phys. Lett. 2021, 763, 138226.

11. Reimers, J. R., A Practical Method for the Use of Curvilinear Coordinates in Calculations of Normal-mode-projected Displacements and Duschinsky Rotation Matrices for Large Molecules. J. Chem. Phys. 2001, 115, 9103-9109.

12. Gao, X.; Bai, S.; Fazzi, D.; Niehaus, T.; Barbatti, M.; Thiel, W., Evaluation of Spin-Orbit Couplings with Linear-Response Time-Dependent Density Functional Methods. J. Chem. Theory Comput. 2017, 13, 515-524.

13. Duan, J. X.; Zhou, Y.; Xie, Z. Z.; Sun, T. L.; Cao, J., Incorporating Spin-orbit Effects into Surface Hopping Dynamics Using the Diagonal Representation: A Linear-response Time-dependent Density Functional Theory Implementation with Applications to 2-thiouracil. Phys. Chem. Chem. Phys. 2018, 20, 15445-15454.

14. Huang, Z.; Bin, Z.; Su, R.; Yang, F.; Lan, J.; You, J., Molecular Design of Nondoped OLEDs Based on a Twisted Heptagonal Acceptor: A Delicate Balance between Rigidity and Rotatability. Angew. Chem. Int. Ed. 2020, 59, 9992-9996. 
